\title{
Mecanismos de rabdomiólise com as estatinas
}

\section{Mechanisms of rhabdomyolysis with statins}

Maria Eliane Campos Magalhães

Setor de Hipertensão Arterial e Lípides do Hospital Universitário Pedro Ernesto - Universidade do Estado do Rio de Janeiro - UERJ

A rabdomiólise é incomum, mas é o efeito adverso mais sério observado na terapia hipolipemiante com estatinas. A ocorrência de rabdomiólise fatal reportada nos Estados Unidos desde a introdução das estatinas no mercado, na década de 1980 , foi muito rara $(0,15$ casos por milhão de pacientes tratados por ano). Entretanto, a miopatia, definida como: sintomas musculares associados com elevações da CK; é muito mais comum (1\%-5\%). Os mecanismos de miopatia mediada por estatinas não estão totalmente compreendidos. Várias hipóteses têm sido propostas: diminuição dos níveis celulares de isoprenóides e ubiquinona, incremento de apoptose, mudanças nos canais de cloro diminuindo a hiperpolarização da membrana celular e alterações da permeabilidade da membrana celular. Interação com outras drogas, e alterações metabólicas pré-existentes podem predispor a miopatia.

\section{Palavras-chave}

Rabdomiólise, estatinas
Rhabdomyolysis is uncommon, but is a serious sideeffect observed following statin use. The occurrence of fatal rhabdomyolysis reported in the USA since the introduction of statins in the market in the late eighties was very rare $(0,15$ cases per million patients treated per year). However, myopathy, defined as muscle symptoms associated with CK elevation is much more common (1-5\%). Mechanism of statin-induced myopathy is not fully understood. Several hypotheses have been proposed: decreased cellular levels of isoprenoids and ubiquinone, increased rates of apoptosis, changes in chloride channels impairing cellmembrane hyperpolarization, and changes in the cellmembrane permeability. Interactions with other drugs, and preexisting metabolic alterations may predispose to myopathy.

\section{KEY WORDS}

Rhabdomyolysis, statins

\section{INTRODUÇÃO}

Os inibidores da hidroximetelglutaril-coenzima-A ( $H M G-C o A)$ redutase, também conhecidos como estatinas, são o grupo de fármacos mais potentes e eficazes para reduzir o LDL colesterol. Desde que foram aprovadas para uso no tratamento da hipercolesterolemia pelo FDA em 1987, diversos estudos clínicos vêm demonstrando que esses medicamentos são capazes de reduzir eventos cardiovasculares, quer na prevenção primária quer na prevenção secundária da doença arterial coronariana (DAC) ${ }^{1}$.

De modo geral, são muito bem tolerados, porém alguns pacientes podem desenvolver toxicidade hepática e/ou muscular, em graus variados. Com relação aos efeitos tóxicos sobre a musculatura esquelética, a complicação mais séria observada com o uso dessas medicações é a rabdomiólise. Felizmente é rara, sendo reportada uma incidência de casos fatais da ordem de 0,15 mortes por 1.000.000 de prescrições, enquanto a freqüência de acometimento muscular menos sério, como a miopatia pode afetar cerca de 1 a $5 \%$ dos pacientes ${ }^{2}$.

O FDA MedWatch Reporting System avaliou 3339 42 casos de rabdomiólise relacionada ao uso de estatina entre janeiro de 1990 e março de 2002. A cerivastatina foi a estatina mais implicada com esta situação ${ }^{3}$. Vale ressaltar, entretanto, que os dados de literatura com relação aos efeitos adversos das estatinas relacionados à musculatura esquelética são confusos, em parte devido à dificuldade em se conceituar adequadamente a rabdomiólise.

Recentemente o American College of Cardiology / American Heart Association definiu quatro formas sindrômicas de acometimento muscular causado pelas estatinas: a miopatia, definida como qualquer desconforto muscular; a mialgia, quando a dor muscular não se acompanha de elevação da enzima creatinofosfoquinase (CPK); a miosite, quando os sintomas musculares se associam com aumento da CPK e a rabdomiólise, quando há importante elevação da CPK (usualmente mais que 10 vezes o limite superior da normalidade) associada com aumento da creatinina sérica, mioglobinúria e insuficiência renal aguda ${ }^{2}$.

O diagnóstico de rabdomiólise também pode ser confirmado por biópsia muscular. 0 aspecto histopatológico se caracteriza por perda do núcleo e das estrias musculares do miócito sem a presença de infiltração de células inflamatórias ${ }^{4}$. 


\section{Farmacologia}

A HMG-CoA redutase é uma enzima que cataliza a conversão da HMG-CoA a ácido mevalônico, precursor do colesterol. Esta enzima é alvo para a intervenção farmacológica porque ela atua na biosíntese do colesterol. Em nível celular, portanto, as estatinas inibem a conversão da HMG-CoA para mevalonato e como conseqüência, a síntese celular do colesterol ${ }^{5}$.

A primeira geração de estatinas, que inclui a lovastatina, a pravastatina e a sinvastatina, é composta por metabólitos fúngicos com estrutura química semelhante. A segunda geração desses fármacos inclui a atorvastatina, a cerivastatina e a fluvastatina. São compostos sintéticos e diferentes estruturalmente. A rosuvastatina é a mais recente estatina disponível no mercado e atua no domínio catalítico da enzima humana. Apesar das diferenças entre todas elas, a maioria apresenta grande ligação com as proteínas plasmáticas, resultando em mínima exposição sistêmica da droga farmacologicamente ativa.

Com exceção da cerivastatina, todas as outras precisam de extensa primeira passagem pelo fígado e a maioria é metabolizada pela enzima citocromo P450 (CYP) 3A4 (exceto a fluvastatina que usa a via P450 2C9 e a pravastatina que é transformada enzimaticamente no citosol do hepatócito e não usa as enzimas CYP) ${ }^{6}$.

\section{MeCanismos de toxicidade MUSCULAR}

O mecanismo exato pelo qual as estatinas podem causar a rabdomiólise não se encontra ainda completamente esclarecido. Diversas hipóteses são possíveis: as estatinas causam depleção de metabólitos intermediários da síntese do colesterol; induzem a apoptose celular e podem causar alterações nos canais de condutância ao cloro dentro dos miócitos ${ }^{6}$.

A inibição da HMG-CoA redutase por essas drogas leva a depleção de metabólitos intermediários formados a partir da síntese do colesterol. Assim, os níveis do ácido mevalônico (farnesol e geranylgeraniol) são reduzidos ${ }^{6}$. Observações recentes com a cerivastatina demonstraram que a administração de mevalonato foi capaz de melhorar os efeitos tóxicos causados pela cerivastatina, sugerindo que as ações deletérias desta droga podiam ser devidas à redução desses compostos intermediários, particularmente o ácido mevalônico, mais do que a ação tóxica direta da mesma ${ }^{7}$.

A coenzima Q10 (CoQ10), a ubiquinona, é um composto isoprenóide que atua na oxidação de nutrientes para produzir ATP, cuja principal função é servir como transportador de elétrons para a mitocôndria ${ }^{8}$. Esta é uma etapa muito importante no desempenho da função do músculo esquelético e cardíaco. Têm-se sugerido que as estatinas causam deficiência intracelular de ubiquinona, mais especificamente bloqueando um intermediário, o farnesil pirofosfato, necessário para a síntese da CoQ10. A redução da CoQ10 resulta em diminuição da fosforilação oxidativa, necessária para o processo normal de respiração celular do miócito ${ }^{8}$.

Estudos de microscopia eletrônica do músculo esquelético de pacientes que recebiam lovastatina revelaram extenso dano mitocondrial ${ }^{9}$. Os autores sugeriram que este dano era secundário à síntese inadequada de CoQ10 e de heme A na membrana mitocondrial interna, com desarranjo subseqüente na produção de energia, levando a morte celular.

England e cols. também observaram que a sinvastatina foi capaz de alterar a síntese da ubiquinona ${ }^{10}$. Estudo conduzido por esses pesquisadores demonstrou a presença de alterações mitocondriais observadas à microscopia eletrônica em pacientes que desenvolveram miopatia. Especula-se se estas alterações podem favorecer e/ou precipitar no músculo esquelético a expressão clínica de uma afecção mitocondrial preexistente. Entretanto, os resultados de outro estudo demonstraram que o tratamento em curto prazo com a sinvastatina não afetou os níveis musculares de ubiquinona ${ }^{8}$. Vinte pacientes hipercolesterolêmicos (colesterol total no período basal variando de 236-397 mg/dL) receberam $20 \mathrm{mg} /$ dia de sinvastatina durante quatro semanas; o grupo controle foi constituído de quinze indivíduos saudáveis (todos homens) que não receberam a medicação do estudo. Os níveis de ubiquinona séricos e no tecido muscular foram determinados no período basal em todos os 35 pacientes e antes do início da medicação no grupo da sinvastatina. Na quarta semana foram coletadas amostras de sangue e músculo no grupo de tratamento. A sinvastatina reduziu os níveis de colesterol total e de LDL em $26 \%$ e $35 \%$, respectivamente, em média. Também foram observados efeitos benéficos nos níveis de triglicérides e de HDL colesterol. Após quatro semanas de tratamento com a sinvastatina os níveis séricos de ubiquinona diminuiram significativamente em $32 \%$ $(p<0.001)$; entretanto os níveis teciduais aumentaram, em média, de $0.060 \mathrm{mg} / \mathrm{gm}$ no período basal para 0.088 $\mathrm{mg} / \mathrm{gm}(+47 \%, \mathrm{p}<0.001)$ no pós-tratamento no grupo da sinvastatina. Esses dados refutam as sugestões prévias de que a sinvastatina diminuiria os níveis musculares de ubiquinona, pelo menos no curto prazo. São necessários estudos adicionais para avaliar os efeitos em longo prazo ${ }^{8}$.

A apoptose, ou morte celular programada, é um mecanismo fundamental na manutenção e remodelação das estruturas tissulares. Quando inapropriadamente ativada, entretanto, a apoptose pode levar a condições patológicas. A atorvastatina, a lovastatina e a sinvastatina aumentam a apoptose de células musculares lisas vasculares, e este é um efeito dose dependente. Os mecanismos pelos quais as estatinas levam a apoptose são ainda pouco claros e mais trabalhos são necessários para demonstrar se um aumento na apoptose poderia causar a miotoxicidade observada com o uso das estatinas ${ }^{11}$.

Outra possível explicação para a toxicidade muscular das estatinas se relaciona com os canais de cloro 
intracelular. Essas estruturas, no músculo, são responsáveis pela hiperpolarização da célula e como conseqüência, do seu relaxamento. Modificações na permeabilidade da membrana celular também podem resultar do bloqueio desse sistema ${ }^{12}$. Tem sido descritas alterações nas propriedades da membrana celular com a utilização desses medicamentos. Algumas estatinas, mais lipofílicas, podem atravessar mais facilmente a membrana celular em função dessas alterações e, por conseguinte, resultar em maior potencial para o desenvolvimento de agressão muscular. Indivíduos que se encontravam em uso de sinvastatina, uma estatina com propriedades lipofílicas, apresentaram elevação dos níveis de CPK, após a realização de exercício físico. Foi sugerido que essas alterações poderiam ser causadas por aumento da permeabilidade da membrana celular muscular à ação da sinvastatina, com possível dano ao miócito e conseqüente liberação de $\mathrm{CPK}^{6}$.

\section{INTERAÇÃO DE DROGAS}

Em um estudo sobre a interação dos inibidores da HMG-CoA redutase com a ciclosporina A em ratos, Smith e cols. ${ }^{13}$ apresentaram dados sugestivos de que a toxicidade para o músculo esquelético é um efeito de classe dos inibidores da HMG-CoA redutase e que a ciclosporina, entre outras drogas, altera a depuração dos inibidres da HMG-CoA redutase, aumentando a exposição tecidual a esses compostos e desse modo, potencializando sua toxicidade. A miopatia ocorre mais freqüentemente quando as estatinas são administradas junto com outros fármacos, também potencialmente miotóxicos ou que permitam a elevação da concentração sérica das estatinas. Assim, pelas razões apontadas, deve-se ter cuidado com a utilização das estatinas em indivíduos portadores de patologias que comprometam a função hepática ou quando houver utilização simultânea das estatinas com outros fármacos que competem com a mesma via metabólica, particularmente com os fibratos. Fatores de risco para miotoxicidade ainda incluem a idade avançada, a insuficiência renal, distúrbios eletrolíticos, doenças inflamatóris dos músculos, hipotireoidismo, cirurgia, trauma e exercícios extenuantes ${ }^{6}$.

\section{ConClusões}

Embora rara, a rabdomiólise pode ser fatal. A identificação de fatores de risco que predisponham para o desenvolvimento de toxicidade muscular antes do início do tratamento com as estatinas é um passo muito importante na prevenção dessas complicações. O ATP III recomenda que a dosagem basal da CPK deve ser feita de rotina em todos os indivíduos e que a dose do medicamento deve ser titulada individualmente.

Quando o nível de CPK for maior que 10 vezes o limite superior do normal esses pacientes devem ser hospitalizados para medidas de suporte e monitorização da função renal.

\section{REFERÊNCIAS}

1. Shepherd J, Cobbe SM and Ford I. Prevention of coronary heart disease with pravastatin in men with hypercholesterolemia. N Engl J Med 1995; 333:1301-7.

2. Pasternak RC, Smith SC, Bairey-Merz CN. ACC/AHA/NHLBI clinical advisory on the use and safety of statins. J Am Coll Cardiol 2002; 40:567-72.

3. Thompson PD, Clarkson $P$ and Karas RH. Statin-Associated Myopathy. JAMA 2003:289:1681-90.

4. Hino I, Akama H, Furuya T. Pravastatin-induced rhabdomyolysis in a patient with mixed connective tissue disease. Arthritis Rheum 1996:39:1259-61.

5. Corsini A, Bellosta S, Baetta R. New insight sinto the pharmacodynamic and pharmacokinetic properties of statins. Pharmacol Ther 1999:84:413-28.

6. Jamal SM, Eisenberg MD and Christopoulos S. Rhabdomyolysis associated with hydroximethylglutaryl-coenzyme A reductase inhibitors. A Heart J 2004:147: 956-65.

7. Gemici G, Toprak A and Oatkay A. Rhabdomyolysis due to cerivastatin monotherapy. Am J Med 2001:110:742.
8. Laaksonen R, Jokelainen K, Sahi T, et al. Decreases in serum ubiquinone concentrations do not result in reduced levels in muscle tissue during short-term simvastatin treatment in. Clin Pharmacol Ther 1995; 57(1): 62-66

9. Manoukian AA, Bhagavan NV, Hayashi T, et al. Rhabdomyolysis secondary to lovastatin therapy. Clin Chemistry 1990; 36(12): 2145-47.

10. England JDF, Viles A, Walsh JC, et al. Muscle side effects associated with simvastatin therapy. M Journal Aust 1990;153(9): 562-63.

11. Knapp AC, Huang J, Starling G. Inhibition of HMGCoA reductase sensitize human smooth muscle cells to FAZ-ligand and cytokineinduced cell death. Atherosclerosis 2000; 152:217-27.

12. Igel $M$, Sudhop $T$, von Bergmann K. Metabolism and drug interation of 3-hydroxy-3-methylglutaryl coenzime A-reductase inhibitors (statins). Eur J Clin Pharmacol 2001: 57:357-64.

13. Smith PF, Eydelloth RS, Grossman SJ, et al. HMG-CoA reductase inhibitor-induced myopathy in the rat: cyclosporine an interaction and mechanism studies. J Pharmacol Exp Ther 1991; 257(3): 1225-1235.

14. Smith JWA, Amelink GJ, de Bruin TWA, et al. Increased human myoglobin and creatine kinase serum levels after standarized exercise during simvastatin treatment. Eur J Clin Invest 1990;20(2 Part 2): A31-A31. 